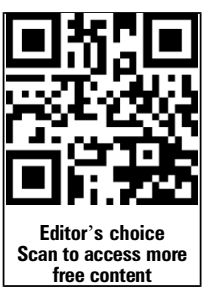

${ }^{1}$ Department of Cardiology, Oslo University Hospital Rikshospitalet, Oslo, Norway ${ }^{2}$ Faculty of Medicine, K. G. Jebsen Cardiac Research Centre and Center for Heart Failure Research, University of Oslo, Oslo, Norway

${ }^{3}$ Institute for Surgical Research, Oslo University Hospital Rikshospitalet, Oslo, Norway ${ }^{4}$ Department of Clinical Science, University of Bergen, Bergen, Norway

${ }^{5}$ Department of Heart Disease, Haukeland University Hospital, Bergen, Norway

${ }^{6}$ Department of Radiology and Nuclear Medicine, Oslo University Hospital Rikshospitalet, Oslo, Norway

\section{Correspondence to}

Dr Kaspar Broch, Department of Cardiology, Oslo University Hospital, Rikshospitalet, Oslo 0027, Norway; kaspar.broch@ ous-hf.no

$\mathrm{KB}$ and SU contributed equally to this study.

Received 17 July 2015 Revised 11 November 2015 Accepted 12 November 2015 Published Online First 9 December 2015

\section{SLinked}

- http://dx.doi.org/10.1136/ heartjnl-2015-308955

\section{CrossMark}

To cite: Broch $\mathrm{K}$, Urheim $\mathrm{S}$, Lønnebakken MT, et al. Heart 2016:102:191-197.

\title{
Controlled release metoprolol for aortic regurgitation: a randomised clinical trial
}

Kaspar Broch, ${ }^{1,2}$ Stig Urheim, ${ }^{1,3}$ Mai Tone Lønnebakken, ${ }^{4,5}$ Wenche Stueflotten,
Richard Massey, ${ }^{1}$ Kristian Fossåa,
${ }^{1}$ Einar Hopp, ${ }^{6}$ Svend Aakhus, ${ }^{1}$ Lars Gullestad ${ }^{1,2}$

ABSTRACT
Objective Chronic aortic regurgitation (AR) creates a

volume load on the left ventricle, which induces adaptive responses. With time, excessive left ventricular (LV) dilatation may precipitate heart failure. $\beta$-adrenergic receptor antagonists ( $\beta$-blockers) are beneficial in patients with heart failure, but their effect in $A R$ is unclear. This trial was designed to evaluate the effect of controlled release metoprolol on LV remodelling in patients with AR.

Methods In this double blind trial, 75 asymptomatic patients aged $44 \pm 14$ years, $89 \%$ males, fulfilling at least two echocardiographic criteria for moderate or severe chronic $A R$, were randomised to receive metoprolol $C R /$ $\mathrm{XL}$ up-titrated to $200 \mathrm{mg} /$ day, or matching placebo. The primary endpoint was LV end diastolic volume, measured by MRI after 6 months of treatment.

Results After 6 months, the difference in the baselineadjusted LV end diastolic volume between patients allocated to metoprolol and those allocated to placebo was $8(95 \% \mathrm{Cl}-8$ to 25$) \mathrm{mL}(\mathrm{p}=0.32)$. The adjusted LV ejection fraction was $2.7(95 \% \mathrm{Cl} 0.1$ to 5.3$)$

percentage points higher in the metoprolol group than in the placebo group $(p=0.04)$. The exercise capacity and peak oxygen consumption did not differ between treatment arms. Serum concentrations of $\mathrm{N}$-terminal proB-type natriuretic peptide were $138(95 \% \mathrm{Cl} 71$ to 205) $\mathrm{pg} / \mathrm{mL}$ higher in the metoprolol group $(p<0.001)$.

There were no serious adverse events in either treatment arm.

Conclusions Treatment with metoprolol of adults with chronic, moderate to severe AR had no effect on LV volumes.

Trial registration number ClinicalTrials.gov Identifier: NCT01157572-results.

\section{INTRODUCTION}

Aortic regurgitation (AR) affects approximately $0.5 \%$ of the population ${ }^{1}$ and is the third most common valvular heart disease in the developed world. ${ }^{12}$ The clinical course of chronic AR is characterised by a prolonged phase of stability, during which the left ventricle adapts to the volume overload and patients remain asymptomatic. ${ }^{3-5}$ However, if left ventricular (LV) dilatation progresses, the probability of death, overt heart failure, or LV dysfunction increases sharply. ${ }^{3-5}$ Today, the only effective treatment is aortic valve replacement or repair. ${ }^{6}$

The role of pharmacological treatment in asymptomatic patients with haemodynamically significant $\mathrm{AR}$ remains unclear. The time to surgical intervention can be delayed by calcium antagonists, $^{7} 8$ ACE inhibitors, ${ }^{9} 10$ and hydralazine. ${ }^{11}$ However, a more recent study did not find any effect of nifedipine or enalapril on either time to surgery or LV size and function. ${ }^{12}$ Treatment with $\beta$-adrenergic receptor antagonists ( $\beta$-blockers) can attenuate or even reverse LV remodelling ${ }^{13}$ and improve survival ${ }^{14} 15$ in heart failure. Nevertheless, $\beta$-blocker therapy has traditionally been discouraged in patients with incipient heart failure due to severe AR. The relative duration of diastole increases when heart rate is reduced, theoretically aggravating the LV volume load in these patients. On the other hand, animal experiments ${ }^{16-19}$ and observational data ${ }^{20}$ suggest that $\beta$-blockers may be cardioprotective in AR. The effect of $\beta$-blockers in AR has not been evaluated in controlled trials in humans. We examined the effect of controlled release metoprolol succinate (metoprolol CR/XL) in asymptomatic patients with chronic, moderate to severe AR, hypothesising that the $\beta$-blockade would reverse $\mathrm{LV}$ remodelling in these patients.

\section{PATIENTS AND METHODS}

This randomised, double blind, placebo-controlled study was designed to assess the effect of metoprolol $\mathrm{CR} / \mathrm{XL}$ on LV size and function in patients with chronic AR (ClinicalTrials.gov Identifier: NCT01157572). It was conducted at two sites in Norway and one in Copenhagen, Denmark. The trial complies with the Declaration of Helsinki and was approved by the appropriate Regional Committees for Medical and Health Research Ethics and the Norwegian Medicines Agency. All patients provided written informed consent. The study was performed in accordance with the Consolidated Standards of Reporting Trials (CONSORT) statement. ${ }^{21}$

\section{Patient population}

Patients aged between 18 and 70 years with asymptomatic, haemodynamically significant $\mathrm{AR}$, an LV ejection fraction (LVEF) $>50 \%$, and an LV end diastolic internal diameter $>5.0 \mathrm{~cm}$ (or an indexed value $>3.0 \mathrm{~cm} / \mathrm{m}^{2}$ ) were eligible. Criteria for exclusion were: symptoms of heart failure; a history of myocardial infarction or symptomatic coronary heart disease; significant aortic stenosis (valvular area $<1.5 \mathrm{~cm}^{2}$ ); additional haemodynamically significant valvular or congenital heart disease; an indication for aortic valve surgery (severe $A R$ in conjunction with either symptoms of heart failure, an LVEF $<50 \%$, or an LV end diastolic/end systolic internal diameter $>7.0 / 5.0 \mathrm{~cm}) ;^{22}$ a second- or 
third-degree atrioventricular block; atrial fibrillation; an intracardiac device; serum creatinine $>250 \mu \mathrm{mol} / \mathrm{L}$; alanine aminotransferase or aspartate aminotransferase $>3$ times the upper limit of normal; any illness or disorder that could severely limit survival; conditions or circumstances likely to lead to poor treatment adherence; and intolerance to metoprolol CR/XL. The concomitant use of vasoactive drugs other than $\beta$-blockers was not an exclusion criterion.

\section{Study procedures}

At baseline, all participants underwent physical examination, blood tests, echocardiography, cardiac MRI, and exercise testing with measurement of peak oxygen consumption. Patients were then randomly assigned to metoprolol $\mathrm{CR} / \mathrm{XL}$ or matching placebo in a 1:1 fashion. The starting dose was $25 \mathrm{mg}$ metoprolol CR/XL or placebo. The dose was doubled every 2 weeks until a target daily dose of $200 \mathrm{mg}$ metoprolol CR/XL or matching placebo was reached, or until side effects precluded a further increase in dosage. Patients were reassessed for safety after 2, 4, 6, 8, and 12 weeks. The physical examination, blood tests, echocardiography, cardiac MRI, and cardiopulmonary exercise test were repeated after 6 months of intervention.

\section{Study outcomes}

Our main objective was to evaluate the effect of metoprolol CR/ XL on LV remodelling in patients with chronic, asymptomatic AR. The primary endpoint was LV end diastolic volume at follow-up as assessed by MRI. Pre-specified secondary outcomes included LV end systolic volume and ejection fraction, serum concentrations of $\mathrm{N}$-terminal pro-B-type natriuretic peptide (NT-proBNP), and peak oxygen consumption. We also assessed functional capacity, quality of life, and safety.

\section{Drug handling, randomisation, and blinding}

Metoprolol CR/XL and matching placebo tablets were provided by the manufacturer (AstraZeneca) and appropriately stored. A randomisation list was produced by computer block randomisation and kept in a sealed envelope until after the last patient had completed follow-up. Study drugs were provided in numbered, otherwise indistinguishable containers and distributed in a double blind fashion by a dedicated study nurse. Study drug adherence was evaluated at 3 and 6 months follow-up based on pill counts of returned, unused study medication. Compliance was considered good if $>80 \%$ of the appropriate number of tablets had been taken.

\section{Imaging}

Cardiac MRI and echocardiography were performed at baseline, before the start of study drug administration, and after 6 months, before study drug discontinuation. All image analyses were performed at Oslo University Hospital, Rikshospitalet. Image analyses were performed by operators blinded to treatment allocation.

\section{Echocardiography}

Echocardiography was performed with Vivid E9 ultrasound scanners (GE Vingmed Ultrasound, Horten, Norway), using phased array transducers. Two dimensional and conventional Doppler measurements were obtained according to current recommendations. $^{23} 24$ The size of the AR was graded from 1 (mild) to 3 (severe) using an integrative approach combining clinical evaluation, valvular morphology, and Doppler and volumetric measurements by echocardiography as recommended in the prevailing guidelines. ${ }^{25}$
MRI

MRIs were acquired with Siemens 1.5 tesla scanners (Siemens Avanto and Siemens Sonata; Siemens Medical Systems, Erlangen, Germany), using a breath-hold, prospectively ECG-triggered, segmented, balanced steady-state free precession gradient-echo cine sequence with minimum echo and repetition times. Slices were $6 \mathrm{~mm}$ thick with a $4 \mathrm{~mm}$ short-axis interslice gap, a spatial resolution of $1.9 \mathrm{~mm} \times 1.3 \mathrm{~mm}$, and a temporal resolution of 30-35 ms. Endocardial borders were traced manually at a PACS (picture archiving and communication system) work station (Sectra Medical Systems AB, Linköping, Sweden). Right ventricle and LV volumes and ejection fractions were calculated by short axis slice summation.

\section{Peak oxygen consumption}

Maximal, symptom-limited exercise testing was performed using an electrically braked bicycle ergometer $(\mathrm{N}=60)$ or a treadmill $(\mathrm{N}=5)$. The bicycle test employed an individualised, stepwise protocol where the workload incrementally increased every minute to reach the age, gender, and weight adjusted expected maximum load after approximately $10 \mathrm{~min}$. For the treadmill test, we used the modified Bruce protocol. Simultaneous gas exchange and haemodynamic monitoring were performed (Cardiovit CS-200, Schiller, Baar, Switzerland and Ganshorn PowerCube, Ganshorn, Niederlauer, Germany). For each individual patient, the same protocol was employed at baseline and follow-up.

\section{Blood sampling and laboratory analysis}

Peripheral blood samples were obtained for routine panel analyses at baseline, 6 weeks, and 6 months. NT-proBNP concentrations were determined by routine methods on MODULAR E 170 analytical platforms (Roche Diagnostics, Mannheim, Germany) by an electrochemiluminescence immunoassay (Roche proBNP II).

\section{Quality of life assessment}

Quality of life was assessed at baseline and after 6 months using two self-reported inventories: the Kansas City Cardiomyopathy Questionnaire (KCCQ) and the EuroQol 5D-3L questionnaire.

\section{Statistics}

To observe an adjusted difference in LV end diastolic volume of at least $15 \mathrm{~mL}$ (representing approximately 10\% of LV volume in a healthy male) between the treatment groups with an $\alpha$ of $5 \%$ and power of $80 \%, 68$ patients (34 in each group) were required, assuming a standard deviation on repeated measurements of $21 \mathrm{~mL}$. The latter figure was based on serial measurements in 50 patients 3 or 6 months after acute myocardial infarction. To compensate for drop-outs, we aimed to enrol 75 patients.

Differences in numerical outcome variables between treatment groups were assessed using analysis of covariance, adjusting for baseline values. Skewed parameters were log-transformed before analysis. The number of adverse events was compared by Poisson regression. All endpoint analyses were performed according to the intention-to-treat principle. Numerical values are presented as mean $\pm S D$ or median (IQR) as appropriate. All statistical analyses were performed in the Statistical Package for Social Sciences V.18 software (SPSS Inc, Chicago, Illinois, USA). Two-sided probability values were considered significant at $\mathrm{p}<0.05$. 


\section{RESULTS}

\section{Patients}

From 24 August 2010 to 30 January 2014, a total of 75 patients were enrolled at the centres in Oslo $(n=69)$, Bergen $(n=5)$, and Copenhagen $(n=1)$. For logistical reasons, patient recruitment was slow at two of the participating centres. However, there were no substantial differences in key characteristics between patients enrolled at the larger centre and those enrolled at the two smaller centres.

The average regurgitation volume by echocardiography was $70 \pm 30 \mathrm{~mL} ; 72 / 75$ patients $(96 \%)$ had a regurgitant volume $>30 \mathrm{~mL}$. All patients fulfilled at least two of current echocardiographic criteria for moderate to severe AR. ${ }^{6}$ Thirty-seven patients were allocated to treatment with metoprolol $\mathrm{CR} / \mathrm{XL}$ and 38 to placebo. The groups were well balanced with respect to baseline characteristics (table 1). One patient in the placebo arm withdrew from the study, and one patient in the metoprolol arm was lost to follow-up. A total of 73 patients were re-evaluated after $175 \pm 17$ days. In one patient assigned to placebo, the quality of the MRI was insufficient for quantification of LV volumes at baseline; therefore, this patient was excluded from primary endpoint analysis (figure 1).

\section{Study drug adherence and dose}

In the metoprolol arm, two patients prematurely discontinued the study drug after 5 and 3 weeks, respectively, due to mild side-effects. Study drug adherence was considered good or excellent in all patients who did not discontinue treatment. The median daily study drug dose after 6 months was $184 \mathrm{mg}$ in patients on metoprolol and tablets equivalent to $200 \mathrm{mg}$

Table 1 Baseline characteristics

\begin{tabular}{|c|c|c|c|c|}
\hline Variable & All patients $(\mathrm{N}=75)$ & Metoprolol ( $\mathrm{N}=37)$ & Placebo $(\mathrm{N}=38)$ & p for difference \\
\hline \multicolumn{5}{|l|}{ Clinical characteristics } \\
\hline Age-years & $44 \pm 14$ & $42 \pm 14$ & $46 \pm 13$ & 0.19 \\
\hline Men-n (\%) & $67(89)$ & $33(89)$ & $34(90)$ & 0.62 \\
\hline Body mass index- $-\mathrm{kg} / \mathrm{m}^{2}$ & $25.8 \pm 3.4$ & $25.1 \pm 3.1$ & $26.5 \pm 3.6$ & 0.08 \\
\hline Systolic blood pressure- $-\mathrm{mm} \mathrm{Hg}$ & $135 \pm 17$ & $133 \pm 18$ & $137 \pm 16$ & 0.29 \\
\hline Diastolic blood pressure- $\mathrm{mm} \mathrm{Hg}$ & $68 \pm 9$ & $66 \pm 9$ & $69 \pm 9$ & 0.22 \\
\hline Resting heart rate- - beats/min & $62 \pm 10$ & $64 \pm 12$ & $60 \pm 7$ & 0.48 \\
\hline Bicuspid aortic valve-n (\%) & $55(73)$ & $28(76)$ & $27(71)$ & 0.62 \\
\hline \multicolumn{5}{|l|}{ Medical history } \\
\hline Smokers-n (\%) & $8(11)$ & $6(16)$ & $2(5)$ & 0.22 \\
\hline History of hypertension-n (\%) & $13(17)$ & $6(16)$ & $7(18)$ & 0.52 \\
\hline Diabetes mellitus-n $(\%)$ & $1(1)$ & $1(3)$ & $0(0)$ & 0.49 \\
\hline Prior stroke/TIA-n (\%) & $2(3)$ & $2(5)$ & $0(0)$ & 0.24 \\
\hline \multicolumn{5}{|l|}{ Baseline medication } \\
\hline ACEI and/or ARB-n (\%) & $12(16)$ & $6(16)$ & $6(16)$ & 0.60 \\
\hline Calcium antagonist-n (\%) & $5(7)$ & $2(5)$ & $3(8)$ & 0.51 \\
\hline Statins-n (\%) & $10(13)$ & $5(13)$ & $5(13)$ & 0.61 \\
\hline Acetylsalicylic acid—n (\%) & $9(12)$ & $7(19)$ & $2(5)$ & 0.07 \\
\hline Other cardiovascular drugs- $\mathrm{n}(\%)$ & $1(1)$ & $1(3)$ & $0(0)$ & 0.51 \\
\hline \multicolumn{5}{|l|}{ Biochemistry } \\
\hline Haemoglobin-g/L & $151 \pm 10$ & $150 \pm 10$ & $152 \pm 10$ & 0.40 \\
\hline Creatinine-mmol/L & $80 \pm 15$ & $81 \pm 16$ & $79 \pm 15$ & 0.52 \\
\hline NT-proBNP—pg/dL & $60(37-136)$ & $59(36-133)$ & $60(39-136)$ & 0.94 \\
\hline \multicolumn{5}{|l|}{ MRI } \\
\hline LV end diastolic volume - $\mathrm{mL}$ & $248 \pm 62$ & $248 \pm 67$ & $247 \pm 56$ & 0.96 \\
\hline LV end diastolic volume index- $-\mathrm{mL} / \mathrm{m}^{2}$ & $119 \pm 23$ & $121 \pm 26$ & $117 \pm 20$ & 0.51 \\
\hline LV ventricular end systolic volume- $-\mathrm{mL}$ & $111 \pm 32$ & $112 \pm 32$ & $110 \pm 32$ & 0.76 \\
\hline LVEF-\% & $55 \pm 7$ & $55 \pm 7$ & $56 \pm 8$ & 0.54 \\
\hline \multicolumn{5}{|l|}{ Echocardiography } \\
\hline LV end diastolic internal diameter- $\mathrm{cm}$ & $6.4 \pm 0.5$ & $6.3 \pm 0.5$ & $6.4 \pm 0.5$ & 0.62 \\
\hline LV end systolic internal diameter-cm & $4.2 \pm 0.4$ & $4.2 \pm 0.4$ & $4.1 \pm 0.4$ & 0.27 \\
\hline LV end diastolic volume- $\mathrm{mL}$ & $242 \pm 45$ & $244 \pm 61$ & $240 \pm 45$ & 0.75 \\
\hline LV stroke volume- $-\mathrm{mL}$ & $159 \pm 33$ & $154 \pm 35$ & $163 \pm 32$ & 0.22 \\
\hline Aortic regurgitant volume- $-\mathrm{mL}$ & $70 \pm 30$ & $66 \pm 29$ & $75 \pm 31$ & 0.17 \\
\hline Aortic regurgitant fraction-\% & $42 \pm 12$ & $39 \pm 12$ & $45 \pm 12$ & 0.06 \\
\hline Vena contracta-mm & $7.6 \pm 1.6$ & $7.5 \pm 1.7$ & $7.7 \pm 1.5$ & 0.46 \\
\hline \multicolumn{5}{|l|}{ Ergospirometry } \\
\hline Peak heart rate-beats/min & $172 \pm 16$ & $176 \pm 15$ & $169 \pm 16$ & 0.052 \\
\hline Peak load-Watts & $238 \pm 65$ & $229 \pm 62$ & $244 \pm 67$ & 0.41 \\
\hline Peak oxygen consumption- $-\mathrm{mL} / \mathrm{kg} / \mathrm{min}$ & $36.1 \pm 9.1$ & $36.0 \pm 8.8$ & $36.2 \pm 9.5$ & 0.93 \\
\hline
\end{tabular}

Baseline characteristics stratified by treatment allocation. Values are presented as mean \pm SD, median (IQR) or number (\%) as appropriate.

ACEI, ACE inhibitor; ARB, angiotensin receptor blocker; LV, left ventricular; LVEF; LV ejection fraction; NT-proBNP, N-terminal pro-B-type natriuretic peptide; TIA, transient ischaemic attack. 
Figure 1 Patient recruitment, randomisation, and follow-up.

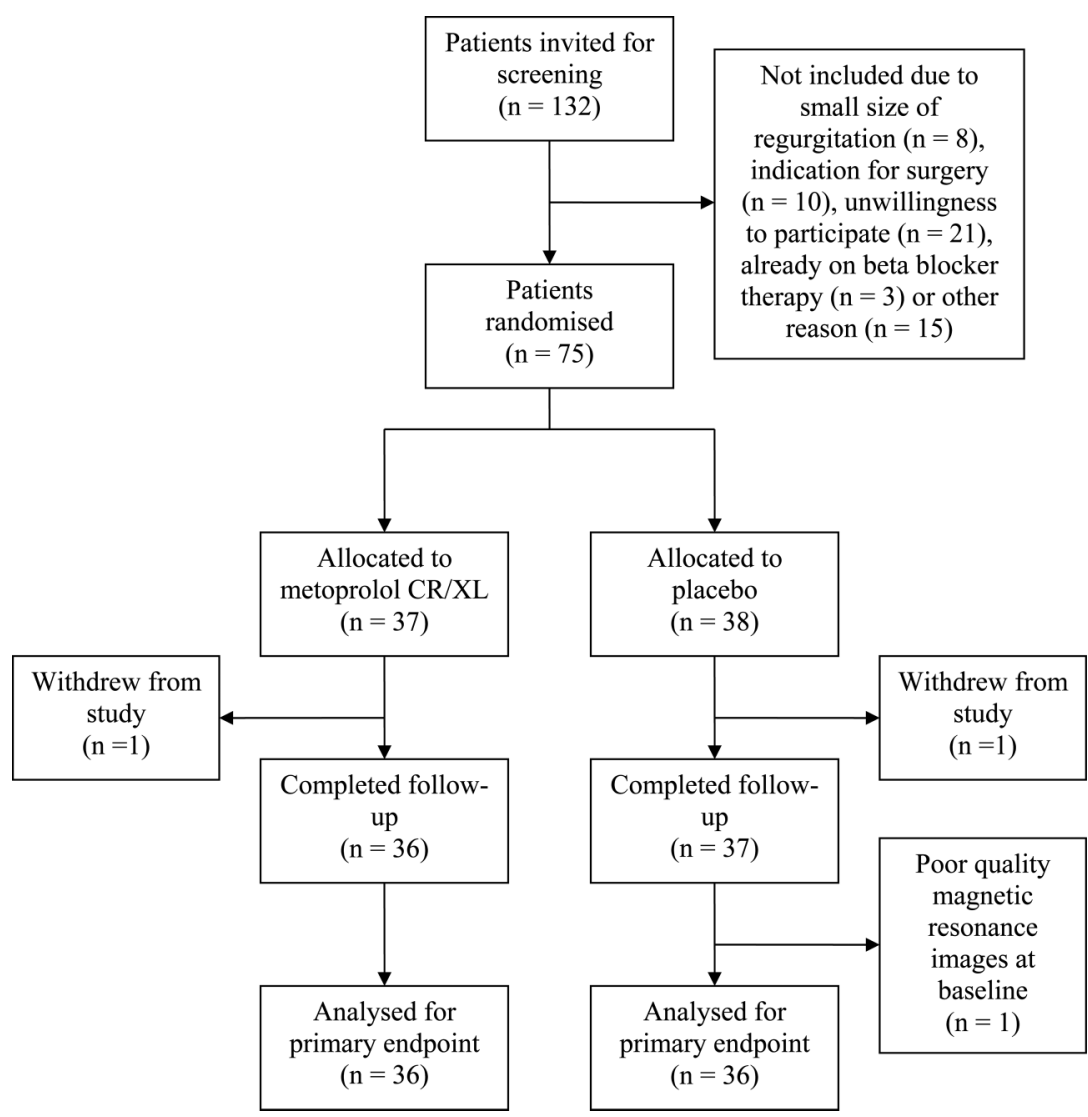

metoprolol in patients on placebo. Correspondingly, the resting heart rate was $8(95 \%$ CI 2 to $15 ; \mathrm{p}=0.02)$ beats $/ \mathrm{min}(\mathrm{bpm})$ and peak heart rate $25(95 \%$ CI 18 to $43 ; \mathrm{p}<0.001) \mathrm{bpm}$ lower in the metoprolol-group.

\section{LV size and function}

Results are summarised in table 2. After 6 months of intervention, there was no difference in baseline-adjusted LV end diastolic volume measured by MRI between patients assigned to metoprolol and those assigned to placebo. This result did not change with adjustment for the baseline AR fraction (adjusted difference 11 (95\% CI -6 to 28$) \mathrm{mL} ; \mathrm{p}=0.21$ ). There was no between-group difference in the baseline-adjusted end systolic volume. The adjusted ejection fraction was 2.7 percentage points higher and the stroke volume $12 \mathrm{~mL}$ larger in patients treated with metoprolol than in patients treated with placebo. Changes in key parameters from baseline to follow-up are illustrated in figure 2 .

\section{Peak oxygen consumption, laboratory results, symptoms, and quality of life}

Exercise testing was performed at baseline and follow-up in 65 patients: 32 patients assigned to metoprolol CR/XL, and 33 patients assigned to placebo. There was no difference in peak oxygen consumption between the $\beta$-blocker and the placebo arm after 6 months of treatment. Baseline-adjusted serum concentrations of NT-proBNP were significantly higher in the metoprolol arm at follow-up. At baseline, all patients were in New York Heart Association (NYHA) functional class I as per design. At the 6 month follow-up, two patients in the metoprolol arm presented as NYHA class II $(p=0.23)$. Quality of life did not change significantly over 6 months and there were no differences in symptoms between the two treatment arms, measured by the EuroQoL visual analogue scale or the KCCQ overall clinical summary score.

\section{Safety and side effects}

Throughout the study period, a total of 54 minor adverse clinical events or side effects were recorded in 36 patients. Twenty-eight events were recorded in 22 patients allocated to metoprolol, and 26 events were recorded in 14 patients allocated to placebo $(p=0.64)$. No serious adverse events or fatalities occurred.

\section{DISCUSSION}

Based on large, randomised trials showing that $\beta$-blocker therapy improves survival and reverses LV remodelling in patients with heart failure, we hypothesised that treatment with controlled release metoprolol would mitigate, or even reverse, LV remodelling in patients with AR. The present study showed that although $\beta$-blocker therapy appears safe in asymptomatic patients with moderate to severe AR, it does not induce a reduction in LV end diastolic or end systolic volume.

$\beta$-blockers prolong diastole, potentially aggravating LV load in AR. On average, the resting heart rate at follow-up was $8 \mathrm{bpm}$ lower in the metoprolol arm. To compensate for this reduction in heart rate, either the LV end diastolic volume would have to increase or the LVEF would have to be augmented to maintain net forward cardiac output. In the $\beta$-blocker group we observed a significant increase in LVEF, although this was not accompanied by a reduction in volume. Our results may reflect a mere physiologic adaptation to the reduction in heart rate. However, the preserved exercise capacity and good tolerability suggest that an amelioration of the sympathetic load may have compensated for the decreased heart rate. Newer imaging techniques, such as speckle-tracking echocardiography, may detect early signs of LV 
Table 2 Results

\begin{tabular}{|c|c|c|c|c|c|c|}
\hline \multirow[b]{2}{*}{ Variable } & \multicolumn{2}{|l|}{ Placebo } & \multicolumn{2}{|l|}{ Metoprolol } & \multirow{2}{*}{$\begin{array}{l}\text { Adjusted difference between } \\
\text { treatment arms at follow-up* } \\
(95 \% \mathrm{Cl})\end{array}$} & \multirow[b]{2}{*}{ p Valuet } \\
\hline & Baseline & Follow-up & Baseline & Follow-up & & \\
\hline Heart rate at rest-beats/min & $67 \pm 12$ & $62 \pm 18$ & $68 \pm 12$ & $55 \pm 17$ & $-8(-15$ to -2$)$ & 0.02 \\
\hline Systolic blood pressure- $-\mathrm{mm} \mathrm{Hg}$ & $136 \pm 16$ & $134 \pm 19$ & $133 \pm 18$ & $124 \pm 17$ & $-7(-13$ to -1$)$ & 0.01 \\
\hline Diastolic blood pressure- $-\mathrm{mm} \mathrm{Hg}$ & $67 \pm 7$ & $67 \pm 6$ & $66 \pm 9$ & $58 \pm 9$ & $-8(-11$ to -6$)$ & $<0.001$ \\
\hline \multicolumn{7}{|l|}{ LV size and function } \\
\hline LV end diastolic volume (MRI)—mL & $246 \pm 57$ & $256 \pm 51$ & $251 \pm 69$ & $267 \pm 87$ & $8(-8$ to 25$)$ & 0.32 \\
\hline LV end systolic volume (MRI)—mL & $110 \pm 33$ & $117 \pm 34$ & $113 \pm 32$ & $117 \pm 46$ & $-4(-14$ to 6$)$ & 0.44 \\
\hline LVEF (MRI)- $\%$ & $55 \pm 8$ & $55 \pm 7$ & $55 \pm 7$ & $57 \pm 7$ & $2.7(0.1$ to 5.3$)$ & 0.04 \\
\hline LV stroke volume (MRI)—mL & $137 \pm 35$ & $139 \pm 28$ & $137 \pm 44$ & $151 \pm 48$ & $12(0$ to 23$)$ & 0.04 \\
\hline Aortic regurgitation fraction (MRI)—\% & $32 \pm 12$ & $37 \pm 18$ & $35 \pm 10$ & $36 \pm 10$ & $-4(-9$ to 1$)$ & 0.08 \\
\hline \multicolumn{7}{|l|}{ Exercise testing } \\
\hline Peak oxygen consumption- $\mathrm{mL} / \mathrm{kg} / \mathrm{min}$ & $35.2 \pm 9.0$ & $36.6 \pm 9.9$ & $35.7 \pm 8.6$ & $34.9 \pm 8.6$ & $-2.0(-4.2$ to 0.2$)$ & 0.08 \\
\hline Peak work-Watts & $237 \pm 63$ & $241 \pm 62$ & $230 \pm 63$ & $229 \pm 62$ & $-6(-15$ to 3$)$ & 0.17 \\
\hline Peak heart rate at exercise-beats/min & $169 \pm 16$ & $168 \pm 16$ & $175 \pm 15$ & $147 \pm 23$ & $-25(-33$ to -18$)$ & $<0.001$ \\
\hline \multicolumn{7}{|l|}{ Natriuretic peptides } \\
\hline NT-proBNP-pg/mL & $62(39-136)$ & $58(42-131)$ & $66(36-138)$ & $142(93-314)$ & 138 (71 to 205$) \ddagger$ & $<0.001$ \\
\hline \multicolumn{7}{|l|}{ Quality of life } \\
\hline EuroQoL visual analogue scale & $82 \pm 11$ & $82 \pm 16$ & $84 \pm 9$ & $85 \pm 7$ & $1(-3$ to 6$)$ & 0.70 \\
\hline KCCQ overall clinical summary score & $98(88-100)$ & $96(91-100)$ & $98(93-100)$ & $98(95-100)$ & $0.4(-2.2$ to 2.8$) \dagger$ & 0.78 \\
\hline
\end{tabular}

dysfunction. However, alterations in heart rate and loading conditions may influence these measurements, and MRI remains the gold standard for assessing LV remodelling.

In healthy volunteers, peak oxygen uptake is reduced by shortterm administration of $\beta$-blockers. ${ }^{26}$ On the other hand, longterm $\beta$-blocker therapy in patients with heart failure improves functional capacity, but has a neutral effect on peak oxygen consumption. ${ }^{27}$ In these patients, the negative chronotropic effect of $\beta$-blockers is balanced by reverse LV remodelling. We observed a substantial reduction in the peak heart rate in the active treatment arm, whereas exercise capacity and peak oxygen consumption remained unchanged. The two patients presenting in NYHA functional class II at follow-up had a combination of dyspnoea and fatigue often observed after the initiation of $\beta$-blocker treatment. Both had unchanged exercise capacity, and their symptoms resolved after discontinuing metoprolol.

Serum concentrations of NT-proBNP were significantly higher in the metoprolol arm than in the placebo arm at
Figure 2 Changes in main outcome variables from baseline to follow-up. Panels show the mean (whiskers 5$95 \%$ interval) changes in (A) left ventricular end diastolic volume (LVEDV), (B) left ventricular end systolic volume (LVESV), (C) left ventricular ejection fraction (LVEF), and (D) $\mathrm{N}$-terminal pro-B-type natriuretic peptide (NT-proBNP) in patients allocated to metoprolol and placebo.
A
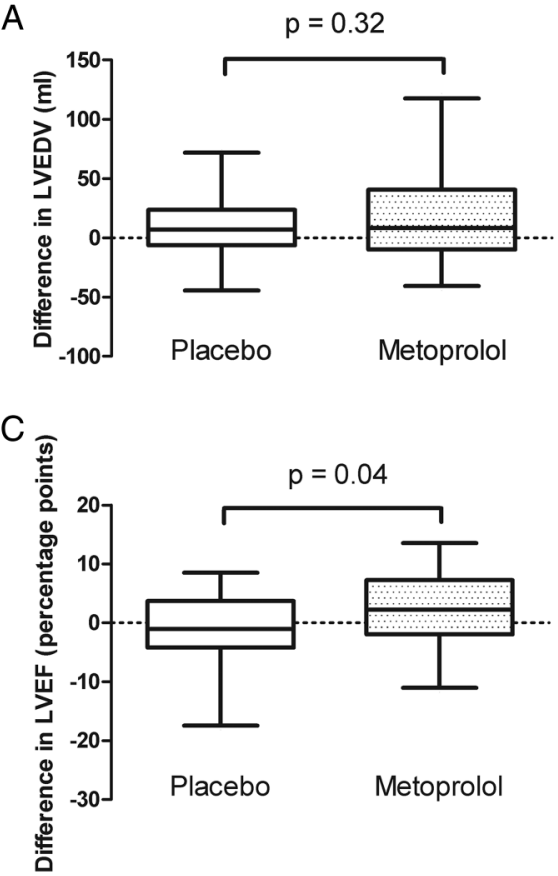

B

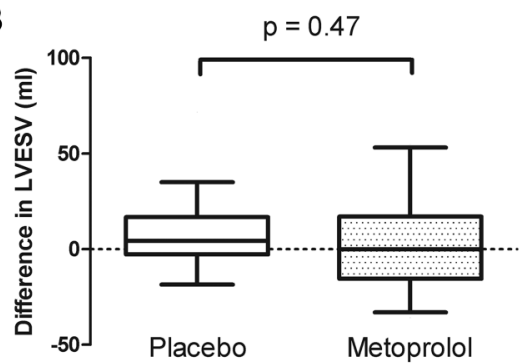

D

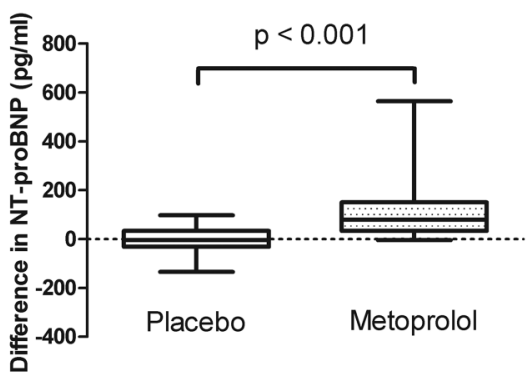


follow-up. Elevated values of natriuretic peptides are associated with adverse outcomes in patients with AR. ${ }^{28}$ However, it remains unclear whether the elevation in NT-proBNP in our patients can be attributed to adverse myocardial stress or just reflects the increased time in diastole. In patients with mild, stable heart failure, the introduction of metoprolol causes a rise in plasma $\mathrm{BNP} / \mathrm{NT}$-proBNP that is unrelated to deterioration in clinical status. $^{29}$

The left ventricle responds to the volume load of chronic AR with a series of compensatory mechanisms, including an increase in end diastolic volume. ${ }^{30}$ However, when compensatory mechanisms are exhausted, heart failure may ensue. Increased sympathetic drive leads to adrenergic receptor down regulation and desensitising, alterations in the myofibrillar protein composition, and subendocardial fibrosis. ${ }^{16-19}$ In the failing heart, $\beta$-blockers can improve myocardial performance by partly reversing these maladaptive biological changes. ${ }^{16} 18$ If the dilatation is primarily adaptive, $\beta$-blocker treatment might not have this effect on LV cavity size. However, this does not mean that $\beta$-blockers cannot prevent the subsequent transition to heart failure.

Several animal studies have shown a beneficial effect of $\beta$-blockade in AR. In a murine model of severe AR, Plante and colleagues showed that after 180 days, the end systolic diameter was smaller and the LVEF higher in rats treated with metoprolol compared with untreated animals, whereas the end diastolic diameter was not significantly different. ${ }^{17}$ Similar results were found by Zendaoui and co-workers. ${ }^{19}$ These results suggest that $\beta$-blocker therapy does indeed protect the LV in AR. Results from a large observational study suggest that $\beta$-blocker therapy confers a survival benefit in human patients with $\mathrm{AR}^{20}$ However, more than two thirds of the patients in this observational study had manifest heart failure, the patients were older and had more comorbidities than our patients, and the LV size was generally smaller.

A treatment time of 6 months is too short to evaluate the effect on harder endpoints, such as the time to develop symptoms, surgery, or death. However, LV reverse remodelling has occurred shortly after the initiation of $\beta$-blockade in other patient groups. In the Metoprolol CR/XL Randomised Intervention Trial in Congestive Heart Failure (MERIT-HF) trial, a $16 \%$ reduction in $\mathrm{LV}$ end diastolic volume was observed after 6 months of $\beta$-blocker therapy. ${ }^{13}$ We calculated sample size based on the assumption that $\beta$-blocker therapy would induce a modest reduction in $\mathrm{LV}$ end diastolic volumes of $15 \mathrm{~mL}$ over 6 months. This is the first controlled trial investigating $\beta$-blocker therapy in chronic AR; therefore, we do not know the optimal dose (if any) in these patients. We aimed for the same dose that has been effective in heart failure trials, and the mean final dose was similar to that obtained in the MERIT-HF trial. ${ }^{15}$ Since a reduction in heart rate in $\mathrm{AR}$ is accompanied by an aggravation of LV load, the dose of metoprolol used to treat our patients may have been higher than optimal.

Asymptomatic patients with severe AR are at risk of sudden death. ${ }^{3} 5$ In heart failure, $\beta$-blockers prevent not only adverse remodelling, but also sudden death from arrhythmia. ${ }^{15}$ Thus, even if $\beta$-blockers do not seem to reverse LV dilatation in patients with AR, they might reduce mortality. ${ }^{18}$ Our trial was not designed to assess clinical endpoints such as mortality or time to aortic valve surgery. The rate of progression to heart failure is low in patients with chronic AR. A large number of study patients would be required to assess the effect of $\beta$-blockers on clinical endpoints. A harmful effect of $\beta$-blocker therapy in AR could not be excluded based on previous trials.

\section{Limitations}

The number of patients was limited and the time span did not allow for assessment of hard clinical endpoints or a potential alleviation (but not reversal) of the slowly progressive LV enlargement observed in AR. Our patients were predominantly male and a large proportion had bicuspid valves. Furthermore, all our patients were asymptomatic and had no significant additional cardiovascular diseases. Care should be taken when extrapolating our results to other patient groups with AR.

\section{CONCLUSION}

Treatment with metoprolol CR/XL for 6 months did not reduce $\mathrm{LV}$ end diastolic volume in asymptomatic patients with chronic AR. Treatment was well tolerated and no serious adverse effects were observed. Our results do not support the use of $\beta$-blocker therapy in patients with moderate to severe AR.

\section{Key messages}

What is already known on this subject?

In patients with aortic regurgitation (AR), excessive left ventricular dilatation may precipitate heart failure unless aortic valve surgery is performed. Treatment with $\beta$-adrenergic receptor antagonists ( $\beta$-blockers) induces reverse remodelling in patients with heart failure, but the effect of $\beta$-blocker therapy in incipient heart failure due to $A R$ is unclear.

\section{What might this study add?}

This randomised, double blind, placebo-controlled trial suggests that medium-term treatment with controlled release metoprolol does not reverse left ventricular remodelling in patients with moderate to severe chronic AR. Treatment with $\beta$ receptor antagonists in these patients does not seem to be associated with serious adverse effects.

How might this impact on clinical practice? Large scale, multinational trials with clinical endpoints would be required to establish more clearly the role of $\beta$ receptor antagonists in chronic AR.

Acknowledgements We would like to thank Doctors Kristian Eskesen, Jan Glasøe, Per Sirnes, Erik Gjertsen, Jan Hysing, Morten Grundtvig, Gunnar Smith, Kjetil Steine and Turid Apelland for their help in patient recruitment. We would also like to thank Doctor Kathrine Rydén Suther, Professor Terje H Larsen, and study Nurse Synnøve Ygre Hauge for their invaluable help in data acquisition.

Contributors All named authors have contributed to the manuscript in compliance with the Uniform Requirements for Manuscripts Submitted to Biomedical Journals. All authors have read and approved the final version of the manuscript. KB: data acquisition, data analysis and interpretation, drafting of the manuscript and approval of final version. SU: study conception and design, data acquisition, data analysis and interpretation, drafting of the manuscript and approval of final version. MTL, RM, KF and EH: data acquisition, analysis and interpretation, critical revision of the manuscript for important intellectual content and approval of final version. WS: data acquisition and analysis, critical revision of the manuscript for important intellectual content and approval of final version. SA and LG: study conception and design, data interpretation, critical revision of the manuscript for important intellectual content and approval of final version.

Funding This work was supported by unrestricted grants provided by the South-East Norway regional health authority (Oslo, Norway) and by the Norwegian ExtraFoundation for Health and Rehabilitation through EXTRA funds (Oslo, Norway). AstraZeneca provided the active drug and placebo tablets used in the study.

Competing interests None declared.

\section{Patient consent Obtained.}

Ethics approval This study was approved by the Regional Committee for Medical and Health Research Ethics (REC South-East) and the Norwegian Medicines Agency. 
Provenance and peer review Not commissioned; externally peer reviewed.

Data sharing statement The study protocol and additional data are available from the corresponding author on request.

\section{REFERENCES}

1 Nkomo VT, Gardin JM, Skelton TN, et al. Burden of valvular heart diseases: a population-based study. Lancet 2006;368:1005-11.

2 lung $B$, Baron $G$, Butchart EG, et al. A prospective survey of patients with valvular heart disease in Europe: The Euro Heart Survey on Valvular Heart Disease. Eur Heart J 2003;24:1231-43.

3 Bonow RO, Lakatos E, Maron BJ, et al. Serial long-term assessment of the natural history of asymptomatic patients with chronic aortic regurgitation and normal left ventricular systolic function. Circulation 1991;84:1625-35.

4 Tornos MP, Olona M, Permanyer-Miralda G, et al. Clinical outcome of severe asymptomatic chronic aortic regurgitation: a long-term prospective follow-up study. Am Heart J 1995;130:333-9.

5 Dujardin KS, Enriquez-Sarano M, Schaff HV, et al. Mortality and morbidity of aortic regurgitation in clinical practice. A long-term follow-up study. Circulation 1999;99:1851-7.

6 Nishimura RA, Otto CM, Bonow RO, et al. 2014 AHA/ACC guideline for the management of patients with valvular heart disease: executive summary: a report of the American College of Cardiology/American Heart Association Task Force on Practice Guidelines. J Am Coll Cardiol 2014;63:2438-88.

7 Scognamiglio R, Rahimtoola SH, Fasoli G, et al. Nifedipine in asymptomatic patients with severe aortic regurgitation and normal left ventricular function. N Engl J Med 1994;331:689-94.

8 Sondergaard $\mathrm{L}$, Aldershvile J, Hildebrandt $\mathrm{P}$, et al. Vasodilatation with felodipine in chronic asymptomatic aortic regurgitation. Am Heart J 2000;139:667-74.

9 Lin M, Chiang HT, Lin SL, et al. Vasodilator therapy in chronic asymptomatic aortic regurgitation: enalapril versus hydralazine therapy. J Am Coll Cardiol 1994:24:1046-53.

10 Alehan D, Ozkutlu S. Beneficial effects of 1-year captopril therapy in children with chronic aortic regurgitation who have no symptoms. Am Heart J 1998;135:598-603.

11 Greenberg B, Massie B, Bristow JD, et al. Long-term vasodilator therapy of chronic aortic insufficiency. A randomized double-blinded, placebo-controlled clinical trial. Circulation 1988:78:92-103.

12 Evangelista A, Tornos $\mathrm{P}$, Sambola A, et al. Long-term vasodilator therapy in patients with severe aortic regurgitation. N Engl J Med 2005;353:1342-9.

13 Groenning BA, Nilsson JC, Sondergaard L, et al. Antiremodeling effects on the left ventricle during beta-blockade with metoprolol in the treatment of chronic heart failure. J Am Coll Cardiol 2000;36:2072-80.
14 [No authors listed]. The Cardiac Insufficiency Bisoprolol Study II (CIBIS-II): a randomised trial. Lancet 1999;353:9-13.

15 [No authors listed]. Effect of metoprolol CR/XL in chronic heart failure: Metoprolol $\mathrm{CR} / \mathrm{XL}$ Randomised Intervention Trial in Congestive Heart Failure (MERIT-HF). Lancet 1999;353:2001-7.

16 Suzuki M, Yoshikawa T, Wainai Y, et al. Beta-blockade prevents ventricular failure following aortic regurgitation in rabbits. Cardiology 1997;88:418-24.

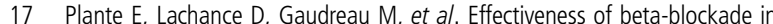
experimental chronic aortic regurgitation. Circulation 2004;110:1477-83.

18 Plante $\mathrm{E}$, Lachance $\mathrm{D}$, Champetier $\mathrm{S}$, et al. Benefits of long-term beta-blockade in experimental chronic aortic regurgitation. Am J Physiol Heart Circ Physiol 2008;294: H1888-95.

19 Zendaoui A, Lachance $D$, Roussel $E$, et al. Usefulness of carvedilol in the treatment of chronic aortic valve regurgitation. Circ Heart Fail 2011;4:207-13.

20 Sampat $U$, Varadarajan $P$, Turk $R$, et al. Effect of beta-blocker therapy on survival in patients with severe aortic regurgitation results from a cohort of 756 patients. J Am Coll Cardiol 2009;54:452-7.

21 Schulz KF, Altman DG, Moher D. CONSORT 2010 statement: updated guidelines for reporting parallel group randomised trials. BMC Med 2010;8:18.

22 Vahanian A, Alfieri 0 , Andreotti F, et al. Guidelines on the management of valvular heart disease (version 2012). Eur Heart J 2012;33:2451-96.

23 Lang RM, Bierig M, Devereux RB, et al. Recommendations for chamber quantification. Eur J Echocardiogr 2006;7:79-108

24 Galderisi M, Henein MY, D'hooge J, et al. Recommendations of the European Association of Echocardiography: how to use echo-Doppler in clinical trials: different modalities for different purposes. Eur J Echocardiogr 2011;12:339-53.

25 Vahanian A, Baumgartner $\mathrm{H}, \mathrm{Bax} J$, et al. Guidelines on the management of valvular heart disease: the task force on the management of valvular heart disease of the European Society of Cardiology. Eur Heart J 2007;28:230-68.

26 Van Baak MA. Beta-adrenoceptor blockade and exercise. An update. Sports Med 1988;5:209-25.

27 Abdulla J, Kober L, Christensen E, et al. Effect of beta-blocker therapy on functional status in patients with heart failure-a meta-analysis. Eur J Heart Fail 2006;8:522-31.

28 Pizarro R, Bazzino 00, Oberti PF, et al. Prospective validation of the prognostic usefulness of B-type natriuretic peptide in asymptomatic patients with chronic severe aortic regurgitation. J Am Coll Cardiol 2011;58:1705-14.

29 Davis ME, Richards AM, Nicholls MG, et al. Introduction of metoprolol increases plasma B-type cardiac natriuretic peptides in mild, stable heart failure. Circulation 2006;113:977-85.

30 Bekeredjian R, Grayburn PA. Valvular heart disease: aortic regurgitation. Circulation 2005;112:125-34. 